\title{
A Liquid Chromatography Assay for the Simultaneous Quantification of Piperacillin and Ciprofloxacin in Human Plasma and Dialysate in Critically Ill Patients Undergoing Continuous Renal Replacement Therapy
}

\author{
Florian Scheer*, Irene Krämer \\ Pharmacy Department, Johannes Gutenberg University Medical Center, Mainz, Germany \\ Email: ${ }^{*}$ florian.scheer@gmail.com
}

Received 22 April 2014; revised 25 May 2014; accepted 15 June 2014

Copyright (C) 2014 by authors and Scientific Research Publishing Inc.

This work is licensed under the Creative Commons Attribution International License (CC BY).

http://creativecommons.org/licenses/by/4.0/

(c) (1) Open Access

\begin{abstract}
Piperacillin/tazobactam and ciprofloxacin are often used in combination as initial empiric antibiotic therapy in critical ill patients. Especially in patients undergoing continuous renal replacement therapy (CRRT) the pharmacokinetics of antimicrobial agents can be highly variable. In order to avoid under- or overdosage of antibiotics therapeutic drug monitoring (TDM) is highly recommendable. Based on two known HPLC assays for piperacillin a new method in combination with solid phase extraction (SPE) for the simultaneous determination of piperacillin and ciprofloxacin was developed. Method validation was performed according to the EMA guideline on validation of bioanalytical methods. The HPLC column used was a Perfect Bond ODS-HD C18 analytical column (100 mm $\times 4.6 \mathrm{~mm}$ i.d., particle size $5 \mu \mathrm{m})$, equipped with a guard column $(10 \mathrm{~mm} \times 4.6$ $\mathrm{mm}$, particle size $5 \mu \mathrm{m}$ ) containing the same packing material. Detection wavelength was set at $228 \mathrm{~nm}$ for piperacillin and benzylpenicillin was used as internal standard (IS). Ciprofloxacin was determined at two wavelengths $(280 \mathrm{~nm}, 315 \mathrm{~nm})$. This newly developed HPLC method in combination with SPE-extraction allows an accurate, precise, specific and efficient determination of piperacillin and ciprofloxacin in biological matrices. Results allow the calculation of all relevant pharmacokinetic data for critically ill patients undergoing CRRT and the optimization of dosing and TDM.
\end{abstract}




\section{Keywords}

\section{Piperacillin, Ciprofloxacin, Solid Phase Extraction (SPE), HPLC-UV, Continuous Renal Replacement Therapy (CRRT), Therapeutic Drug Monitoring (TDM)}

\section{Introduction}

Piperacillin/tazobactam and ciprofloxacin are often used in combination as initial empiric antibiotic therapy in critical ill intensive care patients [1]. Especially in patients undergoing continuous renal replacement therapy (CRRT) the pharmacokinetics of antimicrobial agents can be highly variable [2]. In order to avoid under- or overdosage of antibiotics therapeutic drug monitoring (TDM) is highly recommendable [3] [4]. TDM is based on the measurement of antimicrobial drug concentrations in the biological matrices. Concentrations of antimicrobial agents are usually determined by HPLC assays with ultraviolet (UV) detection. To our knowledge there are no methods published to determine simultaneously concentrations of piperacillin and ciprofloxacin in plasma or dialysate samples. Based on two known HPLC assays for piperacillin a new method for the simultaneous determination of piperacillin and ciprofloxacin was designed [5] [6]. The aim of this study was to develop a rapid and reproducible HPLC method for the simultaneous determination of piperacillin and ciprofloxacin in human plasma and dialysate samples obtained from critical ill patients undergoing CRRT. In addition the same sample pre-treatment method with solid phase extraction (SPE) should be feasible for both antibiotics. Method validation was performed according to the EMA guideline on validation of bioanalytical methods [7].

\section{Experimental}

\subsection{Reagents and Chemicals}

Licensed drug products of piperacillin sodium (Piperacillin/Tazobactam Stragen 4.5 g, Stragen Pharma GmbH, Germany), ciprofloxacin hydrogen sulfate (Ciprofloxacin Kabi Infusionslösung, Fresenius Kabi Deutschland GmbH, Germany), benzylpenicillin sodium and benzylpenicillin potassium (Penicillin Grünenthal 10 Millionen I.E., Grünenthal GmbH, Germany) were used for method validation and in the treatment of patients. Potassium dihydrogen phosphate, disodium hydrogen phosphate-dodecahydrate and sodium hydroxide were obtained from Merck KGaA (Darmstadt, Germany). Sodium dihydrogen phosphate-dihydrate and HPLC-grade water were purchased from Applichem (Darmstadt, Germany). Acetonitril was purchased from LGC-Promochem (Wesel, Germany). Methanol (HPLC grade) was obtained from Fisher Chemical (Loughborough, UK). Ultrapure water (HPLC grade) was obtained from Applichem (Darmstadt, Germany). Fresh frozen human plasma was obtained from the blood bank of the University Medical Center Mainz (Mainz, Germany).

\subsubsection{Standard Solutions of the Antibiotics}

Standard solutions were prepared with ultrapure water to obtain solutions with a nominal concentration of 1 $\mathrm{mg} / \mathrm{ml}$ for piperacillin and benzylpenicillin (internal standard) either. The standard solutions for ciprofloxacin were prepared to obtain solutions with nominal concentrations of $0.1 \mathrm{mg} / \mathrm{ml}$ and $0.01 \mathrm{mg} / \mathrm{ml}$. An appropriate mixture of the standard solutions with ultrapure water and plasma was used to prepare the calibration standards. Standards were stored at $-20^{\circ} \mathrm{C}$.

\subsubsection{Solid Phase Extraction}

Blood samples were centrifuged with a Heraeus Labofuge 400R Function Line (Heraeus, Hanau, Germany). Plasma samples were vortex mixed by a Vortex IKA Minishaker MS2 (IKA, Wilmington, USA). The SPE system consisted of a Vacuum Manifold Chromabond (Macherey Nagel, Düren, Germany) equipped with Chromabond C18ec 1 ml/100mg cartridges (Macherey Nagel, Düren, Germany).

\subsubsection{Instrumentation}

The HPLC system consisted of a Waters Alliance 2695 separation module linked with a Waters 2998 photodiode array ultraviolet detector (PDA). Data were acquired and integrated by using Waters Empower 2 Software 
version 6.10.01.00 (Waters Eschborn, Germany). The column used was a Perfect Bond ODS-HD C18 analytical column, MZ-Analysentechnik, Mainz, Germany (100 mm $\times 4.6 \mathrm{~mm}$ i.d., particle size $5 \mu \mathrm{m})$, equipped with a guard column $(10 \mathrm{~mm} \times 4.6 \mathrm{~mm}$, particle size $5 \mu \mathrm{m})$ containing the same packing material. The autosampler was programmed to inject a volume of $50 \mu \mathrm{l}$ at a carousel temperature of $4^{\circ} \mathrm{C}$ and a run time of $20 \mathrm{~min}$. Detection wavelength was set at $228 \mathrm{~nm}$ for piperacillin and benzylpenicillin as internal standard (IS). Ciprofloxacin was determined at two wavelengths $(280 \mathrm{~nm}, 315 \mathrm{~nm})$.

\subsection{Methods}

\subsubsection{Solid Phase Extraction}

The antibiotics were extracted from plasma using SPE according to a method reported by Denooz and Charlier [5]. Blood samples were centrifuged and $1 \mathrm{ml}$ aliquots of the plasma samples were spiked with $50 \mu \mathrm{l}$ IS. The samples were diluted with $1 \mathrm{ml}$ ultrapure water and vortex mixed. $1 \mathrm{ml}$ aliquots of the diluted samples were used for SPE on Chromabond C18ec cartridges. The antibiotics were eluted with methanol HPLC grade into HPLC vials, $50 \mu \mathrm{l}$ aliquots were injected for HPLC analysis. Dialysate samples underwent no sample pre-treatment. They were spiked with $50 \mu \mathrm{l}$ IS solution and assayed directly.

\subsubsection{Chromatographic Assay}

In analogy to a published method [6], the mobile phase consisted of acetonitrile and $100 \mathrm{mM}$ phosphate buffer 18/82 (v/v) and was adjusted to $\mathrm{pH}$ 6.1. Chromatographic separation was performed by isocratic elution with a flow rate of $1.5 \mathrm{ml} / \mathrm{min}$. The column temperature was set at $17^{\circ} \mathrm{C}$.

Concentrations of piperacillin and the IS (benzylpenicillin) were determined at $228 \mathrm{~nm}$. Concentrations of ciprofloxacin were determined in duplicate at $280 \mathrm{~nm}$ and $315 \mathrm{~nm}$.

\subsubsection{Pharmacokinetic Data Analysis}

Data were acquired and integrated by using Waters Empower 2 Software. Concentration time curves, stability data, precision and accuracy deviations were calculated with Microsoft Excel. Statistical analysis was carried out with IBM SPSS version 20. The resulting concentrations were used to calculate the pharmacokinetic parameters of piperacillin and ciprofloxacin i.e. $\mathrm{t}_{0.5}, \mathrm{Vd}_{\mathrm{ss}}, \mathrm{AUC}, \mathrm{Cl}_{\text {total }}, \mathrm{Cl}_{\mathrm{CRRT}}$ and $\mathrm{Cl}_{\text {extrarenal }}$. Optimum exposure to piperacillin is expected when serum concentrations are maintained 4 - 5 times higher than the minimal inhibitory concentration (MIC), i.e. above $64 \mathrm{mg} / \mathrm{l}$ over the total dosing interval. Optimum exposure to ciprofloxacin is expected when the ratio (AUIC) of the area under the curve (AUC) and MIC is $\geq 125 \mathrm{~h}$ and the $\mathrm{C}_{\max } / \mathrm{MIC}$ ratio amounts to $\geq 10$.

\subsubsection{Method Validation}

The assay was validated according to the EMA Guideline on validation of bioanalytical methods [7]. Linearity, precision, accuracy, selectivity and stability were tested.

Linearity. Calibration curves were obtained by plotting ratios of analyte area under the curve (AUC) divided by internal standard AUC. Calibration standards were prepared by spiking plasma to obtain concentrations between $30-500 \mathrm{mg} / \mathrm{l}$ for piperacillin (30, 50, 100, 200, 300, 400, $500 \mathrm{mg} / \mathrm{l}$ ) and $0.5-10 \mathrm{mg} / \mathrm{l}$ for ciprofloxacin $(0.5,1,2,4,6,8,10 \mathrm{mg} / \mathrm{l})$. A blank sample and a zero sample (plasma spiked with internal standard only) were prepared in the same manner. Each calibration standard was threefold injected for HPLC analysis.

Precision and accuracy. Precision and accuracy were determined within one day (intraday) and on at least two different days (interday). Four calibration standards of piperacillin (30, 50, 200, $400 \mathrm{mg} / \mathrm{l}$ ) and of ciprofloxacin $(0.5,1,4,8 \mathrm{mg} / \mathrm{l})$ were assayed. Within-run accuracy and between-run accuracy were determined by analyzing five samples of each concentration. For the determination of accuracy, the mean concentration should be within $15 \%$ of the nominal values for the QC samples, except for the lower limit of quantification (LLOQ), which should be within $20 \%$ of the nominal value. For the determination of precision, the CV value should not exceed $15 \%$ for the QC samples, except for the LLOQ, which should not exceed $20 \%$.

Selectivity. Selectivity was assessed by testing peak interference in five samples of blank plasma. The interference of co-medication was not tested. The AUC of possible interferences should not succeed $20 \%$ of the target AUC and $5 \%$ of the internal standard.

Stability. Stability was tested for a low and a high calibration standard. Short-term stability was studied after storage in the autosampler $\left(4^{\circ} \mathrm{C}\right)$ for $24 \mathrm{~h}$ and after two freeze-thaw cycles $\left(-20^{\circ} \mathrm{C}\right)$. The concentration measured 
after a $24 \mathrm{~h}$ storage period should not differ more than $\pm 15 \%$ from the initial concentration. Long-term stability was investigated over a period of two months. The concentration after two months should not deviate more than $\pm 15 \%$ from the initial concentration.

\subsection{Pharmacokinetics in Critically Ill Patients}

The clinical application of the liquid chromatography assay for the simultaneous quantification of piperacillin and ciprofloxacin was studied in plasma samples of a typical patient undergoing continuous venovenous haemofiltration (CVVHD). The patient was treated with piperacillin/tazobactam $4.5 \mathrm{~g}$ three times a day and ciprofloxacin $200 \mathrm{mg}$ two times a day. Over a period of 12 hours the steady state plasma levels of piperacillin and ciprofloxacin blood samples were analyzed. Blood samples were withdrawn into heparinized sample syringes directly before and after the intravenous administration of the antibiotic, and after 0.5, 1, 2, 3, 4, 8, 12 hours. Dialysate samples were collected during this interval whenever the dialysate bags were changed. The pharmacokinetic profiles of both antibiotics were constructed. Concentration time curves were calculated with Microsoft Excel.

\section{Results}

\subsection{Method Development}

Based on two known methods simultaneous quantitative analysis of piperacillin and ciprofloxacin in biological matrices was developed [5] [6]. The new method revealed to be feasible, quick and efficient to be used for TDM of broad spectrum antibiotics in patients undergoing CRRT. Representative chromatograms of piperacillin and ciprofloxacin analyzed in plasma and dialysate samples are shown in Figure 1 and Figure 2.

\subsection{Method Validation}

Statistical analysis of the concentration-response ratios proved in all cases that linear correlation was the best model in the studied concentration range. Correlation coefficients for all calibration curves were higher than 0.997. Linearity of the calibration curve was given up to $500 \mathrm{mg} / \mathrm{l}$ for piperacillin and up to $10 \mathrm{mg} / \mathrm{l}$ for ciprofloxacin. Results of interday precision, intraday precision and accuracy for plasma and dialysate samples are shown in Table 1 and Table 2, respectively. For piperacillin the intra- and interday precision deviation was lower than 7\%. Accuracy ranged from $87 \%$ - 102\% on the different concentration levels. For ciprofloxacin the intra- and interday precision deviation was lower than 9\%. Accuracy ranged from $91 \%-104 \%$ on the different concentrations.

The LOQ corresponded to the lowest standard concentrations used to determine the calibration curves. For piperacillin the LOQ should be lower than $64 \mathrm{mg} / \mathrm{l}$ (four times the minimum inhibitory concentration (MIC) of typical infectious agents $(16 \mathrm{mg} / \mathrm{l})$ ). According to the lowest concentration used to construct the calibration curve the LOQ was set at $30 \mathrm{mg} / \mathrm{l}$. At this concentration the piperacillin-peak was five times higher than the base line signal. For ciprofloxacin the LOQ was set at the MIC of pseudomonas aeruginosa $(0.5 \mathrm{mg} / \mathrm{l})$. At this concentration the ciprofloxacin-peak was five times higher than the base line signal. The LOQ amounted to 5\% of the expected maximal concentration of $10 \mathrm{mg} / \mathrm{l}$ [8].

There were no peak interferences observed in five samples of different blank plasmas.

Piperacillin and ciprofloxacin were stable in plasma-samples over a two months period when stored frozen at $-20^{\circ} \mathrm{C}$ or a one-day period when stored refrigerated. Piperacillin revealed to be stable for two freeze-thawcycles, whereas ciprofloxacin was only stable for one cycle.

Frozen dialysate samples spiked with piperacillin or ciprofloxacin revealed to be stable for two months or 24 hours, respectively. Refrigerated piperacillin and ciprofloxacin samples can be stored for one day. Two freezethaw cycles can be executed with no significant losses.

\subsection{Pharmacokinetics in Critically Ill Patients}

Concentration time curves of piperacillin and ciprofloxacin of a selected patient (male patient, 58 years, $175 \mathrm{~cm}$ high, body weight $69 \mathrm{~kg}$, septic with liver transplant dysfunction) are shown in Figure 3. Because of acute renal failure (ARF) the patient underwent renal replacement therapy with CVVHD (blood flow: $120 \mathrm{ml} / \mathrm{min}$; dialysate flow: $2.2 \mathrm{l} / \mathrm{h}$; ultrafiltration: $100 \mathrm{ml} / \mathrm{h}$ ). 

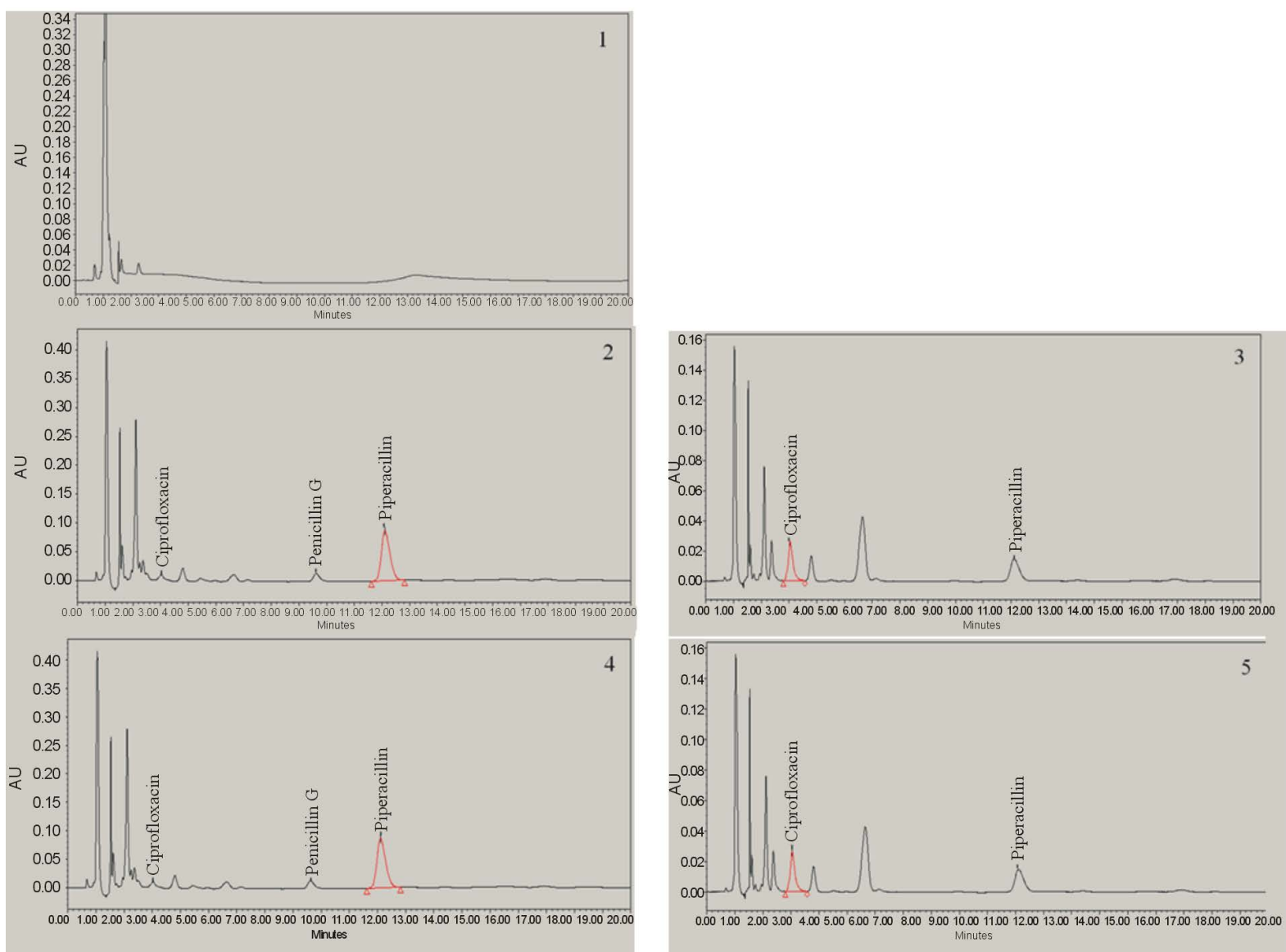

Figure 1. HPLC chromatograms of blank plasma, detection wavelength $228 \mathrm{~nm}$ (1), plasma spiked with piperacillin, ciprofloxacin and benzylpenicillin (IS) detection wavelength $228 \mathrm{~nm}$ (2), plasma spiked with piperacillin, ciprofloxacin and benzylpenicillin (IS), detection wavelength $280 \mathrm{~nm}$ (3), plasma from the patient receiving piperacillin $4000 \mathrm{mg}$ three times a day and ciprofloxacin $200 \mathrm{mg}$ twice a day, detection wavelength $228 \mathrm{~nm}$ (4), detection wavelength $280 \mathrm{~nm}$ (5).
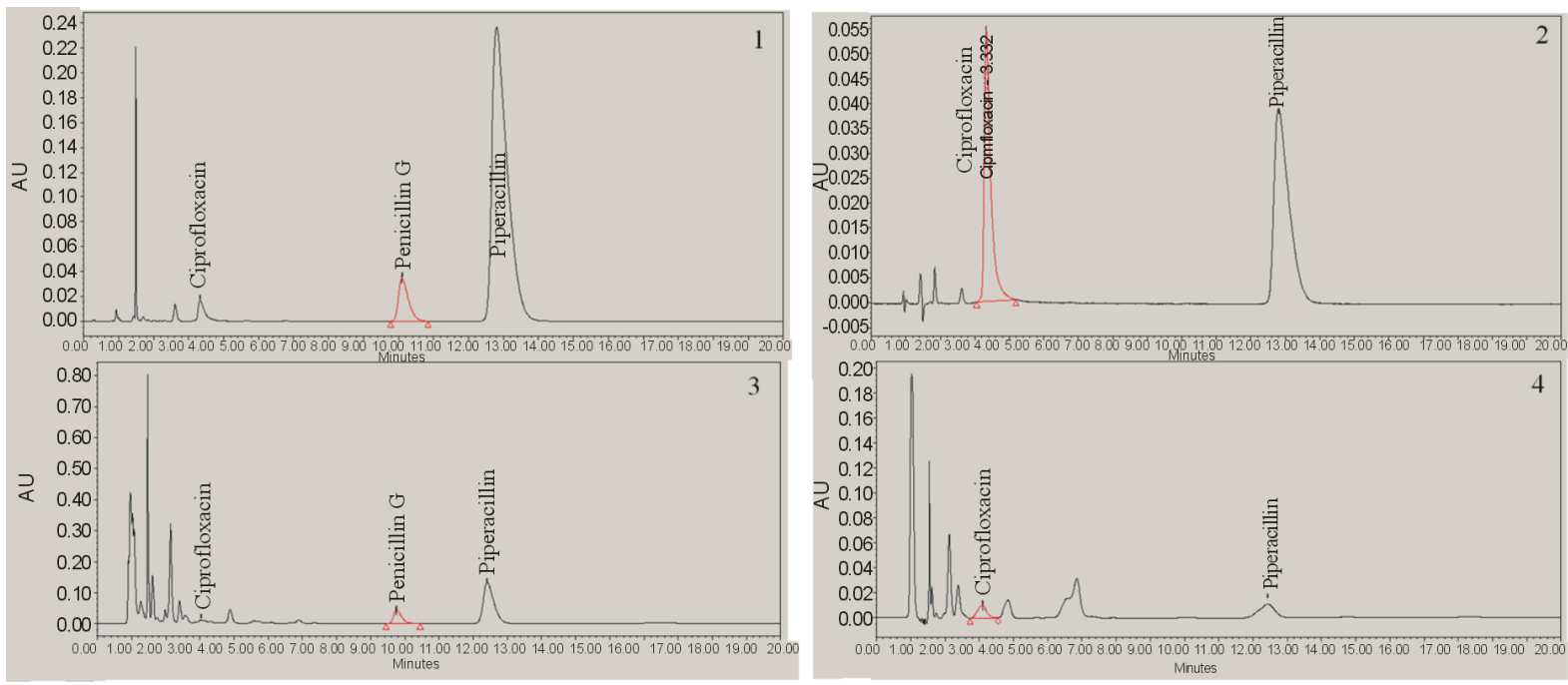

Figure 2. HPLC chromatograms of dialysate spiked with piperacillin, ciprofloxacin and benzylpenicillin (IS), detection wavelength $228 \mathrm{~nm}$ (1), dialysate spiked with piperacillin, ciprofloxacin and benzylpenicillin (IS), detection wavelength $280 \mathrm{~nm}$ (2), dialysate from patient receiving piperacillin $4000 \mathrm{mg}$ three times a day and ciprofloxacin $200 \mathrm{mg}$ twice a day detection wavelength $228 \mathrm{~nm}$ (4), detection wavelength $280 \mathrm{~nm}$ (5). 
Table 1. Precision and accuracy of the HPLC assay of piperacillin and ciprofloxacin in plasma samples.

\begin{tabular}{|c|c|c|c|c|c|c|}
\hline Nominal concentration & Intra-dayprecision $(\mathrm{n}=10)$ & \multirow{2}{*}{$\begin{array}{l}\text { CV } \\
(\%)\end{array}$} & \multirow{2}{*}{$\begin{array}{l}\text { Bias } \\
(\%)\end{array}$} & \multirow{2}{*}{$\begin{array}{c}\text { Inter-dayprecision }(\mathrm{n}=15) \\
\text { Mean measured concentration } \\
\pm \mathrm{SD}(\mathrm{mg} / \mathrm{l})\end{array}$} & \multirow{2}{*}{$\begin{array}{l}\text { CV } \\
(\%)\end{array}$} & \multirow{2}{*}{$\begin{array}{l}\text { Bias } \\
(\%)\end{array}$} \\
\hline$(\mathrm{mg} / \mathrm{l})$ & $\begin{array}{l}\text { Mean measured concentration } \\
\pm \mathrm{SD}(\mathrm{mg} / \mathrm{l})\end{array}$ & & & & & \\
\hline \multicolumn{7}{|l|}{ Piperacillin (228 nm) } \\
\hline 30 & $26.18 \pm 1.26$ & 4.8 & 12.74 & $26.11 \pm 1.08$ & 4.14 & 12.96 \\
\hline 50 & $48.61 \pm 1.6$ & 3.29 & 2.77 & $48.85 \pm 1.44$ & 2.94 & 2.31 \\
\hline 200 & $187.51 \pm 4.49$ & 2.39 & 6.24 & $188.31 \pm 4.33$ & 2.3 & 5.84 \\
\hline 400 & $367.05 \pm 9.64$ & 2.63 & 8.24 & $376.29 \pm 13.07$ & 3.47 & 5.93 \\
\hline \multicolumn{7}{|l|}{ Ciprofloxacin (280 nm) } \\
\hline 0.5 & $0.5 \pm 0.04$ & 8.02 & 0.44 & $0.5 \pm 0.04$ & 8.99 & 0.6 \\
\hline 1 & $1.04 \pm 0.09$ & 8.82 & 3.91 & $1.03 \pm 0.09$ & 8.49 & 2.95 \\
\hline 4 & $3.98 \pm 0.19$ & 4.74 & 0.59 & $3.93 \pm 0.18$ & 4.47 & 1.7 \\
\hline 8 & $7.94 \pm 0.38$ & 4.81 & 0.8 & $7.86 \pm 0.35$ & 4.4 & 1.75 \\
\hline \multicolumn{7}{|l|}{ Ciprofloxacin (315 nm) } \\
\hline 0.5 & $0.5 \pm 0.02$ & 4.95 & 0.37 & $0.5 \pm 0.03$ & 5.05 & 0.96 \\
\hline 1 & $1.04 \pm 0.06$ & 5.72 & 3.88 & $1.03 \pm 0.05$ & 5.1 & 3.04 \\
\hline 4 & $4.08 \pm 0.17$ & 4.05 & 1.87 & $4.03 \pm 0.18$ & 4.41 & 0.69 \\
\hline 8 & $8.11 \pm 0.41$ & 5.11 & 1.35 & $7.97 \pm 0.43$ & 5.37 & 0.43 \\
\hline
\end{tabular}

SD = Standard deviation; CV = Coefficientofvariation.

Table 2. Precision and accuracy of the HPLC assay of piperacillin and ciprofloxacin in dialysate samples.

\begin{tabular}{|c|c|c|c|c|c|c|}
\hline \multirow[b]{2}{*}{ Nominal concentration } & Intra-day $n=10$ & \multirow{2}{*}{$\begin{array}{l}\mathrm{CV} \\
(\%)\end{array}$} & \multirow{2}{*}{$\begin{array}{l}\text { Bias } \\
(\%)\end{array}$} & \multirow{2}{*}{$\begin{array}{c}\text { Inter-day }(\mathrm{n}=15) \\
\text { Mean measured concentration } \\
\pm \mathrm{SD}\end{array}$} & \multirow{2}{*}{$\begin{array}{l}\text { CV } \\
(\%)\end{array}$} & \multirow{2}{*}{$\begin{array}{l}\text { Bias } \\
(\%)\end{array}$} \\
\hline & $\begin{array}{c}\text { Mean measured concentration } \\
\pm \mathrm{SD}\end{array}$ & & & & & \\
\hline \multicolumn{7}{|l|}{ Piperacillin (228 nm) } \\
\hline 30 & $29.68 \pm 0.15$ & 0.5 & 1.07 & $30.47 \pm 1.22$ & 4.00 & 1.57 \\
\hline 50 & $48.93 \pm 0.65$ & 1.33 & 2.13 & $50.68 \pm 2.41$ & 4.75 & 1.36 \\
\hline 200 & $189.88 \pm 2.51$ & 1.32 & 5.06 & $199.21 \pm 12.45$ & 6.25 & 0.4 \\
\hline 400 & $384.86 \pm 7.94$ & 2.06 & 3.78 & $401.29 \pm 23.1$ & 5.76 & 0.32 \\
\hline \multicolumn{7}{|l|}{ Ciprofloxacin (280 nm) } \\
\hline 0.5 & $0.46 \pm 0.02$ & 3.39 & 7.25 & $0.45 \pm 0.02$ & 4.25 & 9.27 \\
\hline 1 & $0.93 \pm 0.02$ & 2.00 & 7.4 & $0.92 \pm 0.02$ & 2.52 & 8.18 \\
\hline 4 & $4.01 \pm 0.14$ & 3.43 & 0.33 & $4.04 \pm 0.13$ & 3.31 & 0.99 \\
\hline 8 & $8.03 \pm 0.21$ & 2.59 & 0.43 & $8.2 \pm 0.32$ & 3.86 & 2.47 \\
\hline
\end{tabular}

$\mathrm{SD}=$ Standard deviation; $\mathrm{CV}=$ Coefficientofvariation .

\section{Discussion}

To the best of our knowledge a simultaneous and quick HPLC method suitable for TDM of $\beta$-lactams and quinolones is not published yet. The HPLC method described here, revealed to be valid for the rapid simultaneous determination of piperacillin and ciprofloxacin concentrations in plasma and dialysate samples. Including 40 min for SPE and 840 min for the HPLC assay (9 plasma samples, 1 blank sample (methanol), 1 zero sample (methanol/internal standard), 3 quality control (QC) samples). The assay took 14 - 15 hours per patient. During the validation study the arithmetic mean of three injections was used to construct the calibration curve. Blank samples were integrated in each HPLC run; QC samples were only integrated randomly because of high costs. In these runs precision and accuracy specifications were fulfilled. There was no carry-over measured in any blank sample. The minimum and maximum concentrations in the biological matrices were expected to fall into 

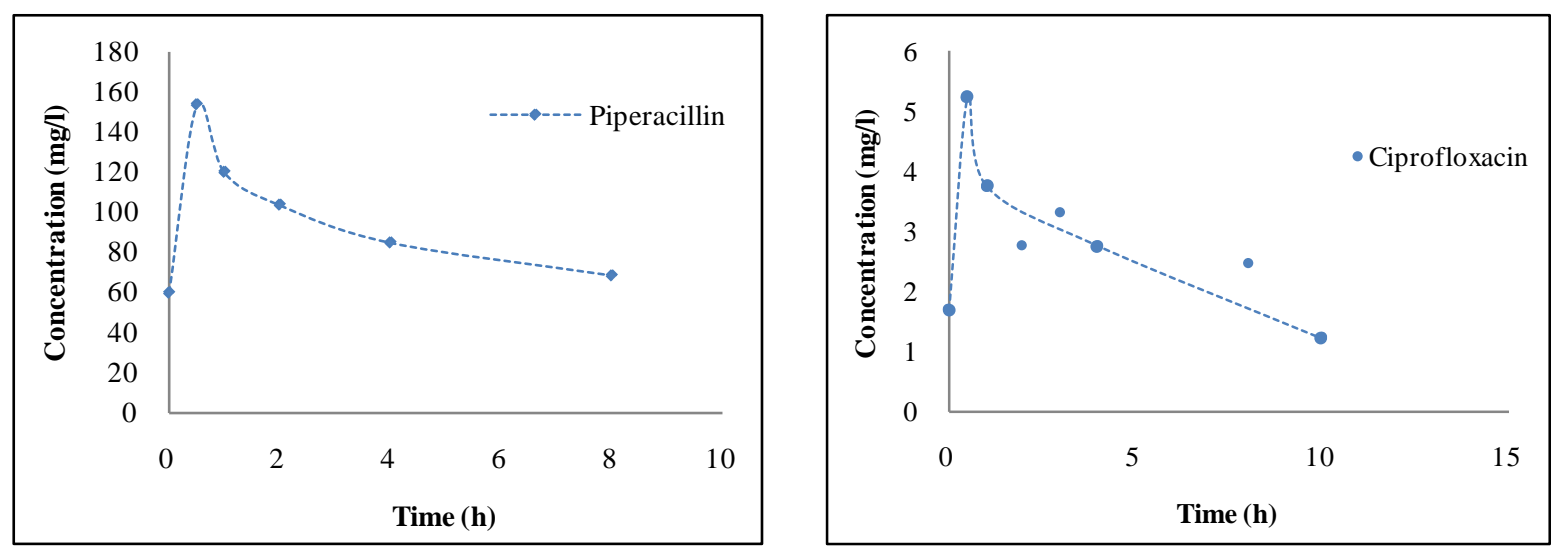

Figure 3. Serum levels of piperacillin and ciprofloxacin over a $12 \mathrm{~h}$ observation period. The patient undergoing CVVHD was medicated with $4000 \mathrm{mg}$ piperacillin/tazobactam and $200 \mathrm{mg}$ ciprofloxacin as intermittent infusion.

the linear range of the calibration curve. In case of piperacillin concentrations should not go below $64 \mathrm{mg} / \mathrm{l}$. In case of ciprofloxacin lower concentrations than $0.5 \mathrm{mg} / \mathrm{l}$ do not have a relevant impact on the resulting AUIC. Because of this insignificant impact and the calibration curves are lines through origin, concentrations lower than the validated range can be extrapolated.

Precision and accuracy of the new HPLC method fulfilled the specifications of the Guideline on validation of bioanalytical methods [7]. However precision and accuracy deviations were higher than those reported with a similar method [6]. This is to be explained by the additional SPE. Plasma samples can be diluted with water without exceeding the precision and accuracy limits of $\pm 15 \%$. According to the supernatant results and other studies plasma samples containing piperacillin and ciprofloxacin can be stored at $-20^{\circ} \mathrm{C}$ for two months [5] [6] [9]. Two freeze thaw cycles are possible for piperacillin samples. Of note ciprofloxacin containing plasma samples should be only frozen and thawed once [9].

Precision and accuracy deviations of the HPLC assay were lower in dialysate samples than in plasma samples. This is to be explained by direct injection of dialysate samples. Dialysate samples containing piperacillin can be stored at $-20^{\circ} \mathrm{C}$ for two months. Dialysate samples containing ciprofloxacin are not stable over a storage period of one month. Polyvalent cations like magnesium in dialysate samples form complexes and thereby reduce the ciprofloxacin concentration [10] [11]. Ciprofloxacin samples are stable over 24 hours when stored refrigerated. Analysis of dialysate samples should be performed directly after the collection period. Otherwise samples should be stored frozen. For both antibiotics two freeze thaw cycles are possible.

The HPLC method is suitable for routine monitoring of the antibiotic plasma levels in critically ill patients undergoing continuous renal replacement therapy. TDM as evaluation and interpretation of plasma or serum concentrations is a method to individualize drug therapy on a pharmacokinetic level. In the first place TDM is used for drugs with a narrow therapeutic range. In the treatment of septic patients antibiotics belong to the most important drugs and the pharmacokinetics of antimicrobial agents in critically ill patients can be highly variable [2]. In antibiotic therapy the therapeutic effects cannot be registered directly and under treatment is not recognized promptly. In order to optimize the outcome of antibiotic therapy in septic patients therapeutic drug monitoring is recommended [3].

With standard equipment like UV detection, an isocratic method and a short runtime the simultaneous determination of typically used antibiotics in a biological and aqueous matrix is possible. One advantage of this method is the simultaneous pre-treatment of ten blood samples. The simultaneous sample pre-treatment allows the SPE and HPLC assay per sample to be performed in about $22 \mathrm{~min}$. The procedure for sample pre-treatment is rapid and inexpensive. Because the IS and samples containing unknown concentrations are handled simultaneously, losses during the SPE pre-treatment are equalized. Sample preparation is easier than in other known methods for the determination of piperacillin [5] [6] [12]-[16] and ciprofloxacin [9] [17]-[23] in plasma and dialysate samples. There the separation of antibiotics from plasma is accomplished by protein precipitation [6] [9] [13] [17] [18] [22], liquid-liquid extraction [15] [20] [21], filtration [23] or solid phase extraction [5] [16] [19]. The external SPE was favored over the solid phase extraction method because of a shorter analysis time per sample. In the latter one piperacillin is separated on the column by column-switching [24]. 
According to the national German guideline the combination of piperacillin and ciprofloxacin is recommended in the treatment of nosocomial septic patients with unknown focus [1]. Corresponding to the clinical guideline piperacillin and ciprofloxacin are often used and were selected for the pharmacokinetic study. The developed analytical method revealed to be suitable to calculate the concentration time curve for the antibiotic substances investigated. In combination with the results of the dialysate samples, all relevant pharmacokinetic data for patients undergoing CRRT can be calculated. This essential basis for optimization of dosing and TDM is given by the newly developed method. Because of the easy sample pre-treatment and the reliability of the assay therapeutic drug monitoring is feasible in clinical practice.

\section{Conclusion}

This newly developed HPLC method in combination with SPE-extraction allows an accurate, precise, specific and efficient determination of piperacillin and ciprofloxacin in biological matrices. Both antibiotics can be analyzed simultaneously in plasma as well as dialysate samples. Results allow the calculation of all relevant pharmacokinetic data for critically ill patients undergoing CRRT and the optimization of dosing and TDM.

\section{Competing Interests}

All authors declare they have no competing interests.

\section{References}

[1] Bodmann, K. and Grabein, B. (2010) Empfehlungen zur kalkulierten parenteralen Initialtherapie bakterieller Erkrankungen bei Erwachsenen.

[2] Trotman, R.L., Williamson, J.C., Shoemaker, D.M. and Salzer, W.L. (2005) Antibiotic Dosing in Critically Ill Adult Patients Receiving Continuous Renal Replacement Therapy. Clinical Infectious Diseases, 41, 1159-1166. http://dx.doi.org/10.1086/444500

[3] Pea, F., Viale, P., Pavan, F. and Furlanut, M. (2007) Pharmacokinetic Considerations for Antimicrobial Therapy in Patients Receiving Renal Replacement Therapy. Clinical Pharmacokinetics, 46, 997-1038. http://dx.doi.org/10.2165/00003088-200746120-00003

[4] Eggimann, P. and Pittet, D. (2001) Infection Control in the ICU. Chest, 120, 2059-2093. http://dx.doi.org/10.1378/chest.120.6.2059

[5] Denooz, R. and Charlier, C. (2008) Simultaneous Determination of Five $\beta$-Lactam Antibiotics (Cefepim, Ceftazidim, Cefuroxim, Meropenem and Piperacillin) in Human Plasma by High-Performance Liquid Chromatography with Ultraviolet Detection. Journal of Chromatography B, 864, 161-167. http://dx.doi.org/10.1016/j.jchromb.2008.01.037

[6] Arzuaga, A., Isla, A., Gascon, A.R., Maynar, J., Martin, A., Solinis, M.A., Toral, D. and Pedraz, J.L. (2005) Quantitation and Stability of Piperacillin and Tazobactam in Plasma and Ultrafiltrate from Patients Undergoing Continuous Venovenous Hemofiltration by HPLC. Biomedical Chromatography, 19, 570-578. http://dx.doi.org/10.1002/bmc.482

[7] EMA and CHMP (2011) Guideline on Bioanalytical Method Validation.

[8] Bayer Schering Pharma (2010) Fachin Formation Ciprobay 400 mg 34.

[9] Watabe, S., Yokoyama, Y., Nakazawa, K., Shinozaki, K., Hiraoka, R., Takeshita, K. and Suzuki, Y. (2010) Simultaneous Measurement of Pazufloxacin, Ciprofloxacin, and Levofloxacin in Human Serum by High-Performance Liquid Chromatography with Fluorescence Detection. Journal of Chromatography B, 878, 1555-1561. http://dx.doi.org/10.1016/j.jchromb.2010.04.012

[10] Steinhilber, D., Schubert-Zsilavecz, M. and Rot, H. (2005) Medizinische Chemie. Deutscher Apotheker Verlag, Stuttgart.

[11] Hubicka, U., Krzek, J. and Walczak, M. (2010) Stability of Ciprofloxacin and Norfloxacin in the Presence and Absence of Metal Ions in Acidic Solution. Pharmaceutical Development and Technology, 15, 532-544. http://dx.doi.org/10.3109/10837450903338379

[12] Augey, V., Grosse, P.Y., Albert, G., Audran, M. and Bressolle, F.J. (1996) High-Performance Liquid Chromatographic Determination of Tazobactam and Piperacillin in Human Plasma and Urine. Journal of Chromatography B: Biomedical Sciences and Applications, 682, 125-136. http://dx.doi.org/10.1016/0378-4347(96)00049-7

[13] Di, G.G., Ferrannini, M., Testore, G.P., Federici, G.and Pastore, A. (2009) High Performance Liquid Chromatographic Determination of Plasma Free and Total Tazobactam and Piperacillin. Journal of Chromatography B, 877, 86-88. http://dx.doi.org/10.1016/j.jchromb.2008.11.010 
[14] Marselos, S.C. and Archontaki, H.A. (2002) Development and Optimization of a Reversed-Phase High-Performance Liquid Chromatographic Method for the Determination of Piperacillin and Tazobactam in Tazocin Injectable Powder. Journal of Pharmaceutical and Biomedical Analysis, 29, 787-793. http://dx.doi.org/10.1016/S0731-7085(02)00129-2

[15] McWhinney, B.C., Wallis, S.C., Hillister, T., Roberts, J.A., Lipman, J. and Ungerer, J.P. (2010) Analysis of 12 BetaLactam Antibiotics in Human Plasma by HPLC with Ultraviolet Detection. Journal of Chromatography B, 878, 20392043. http://dx.doi.org/10.1016/j.jchromb.2010.05.027

[16] Xia, C.H., Xiong, Y.Q. and Wang, G.J. (2007) An Improved High-Performance Liquid Chromatographic Method with a Solid-Phase Extraction for the Determination of Piperacillin and Tazobactam: Application to Pharmacokinetic Study of Different Dosage in Chinese Healthy Volunteers. Biomedical Chromatography, 21, 680-686. http://dx.doi.org/10.1002/bmc.800

[17] Bardot, S., Vest, P., Renard, C. and Renaudeau, C. (2005) Determination of Ciprofloxacin in Human Serum by HighPerformance Liquid Chromatography. Annales de Biologie Clinique (Paris), 63, 513-516.

[18] De, S.J., Boussery, K., Colpaert, K., De, S.P., De, P.P., Decruyenaere, J. and Van Bocxlaer, J. (2009) Pharmacokinetics of Fluoroquinolones in Critical Care Patients: A Bio-Analytical Hplc Method for the Simultaneous Quantification of Ofloxacin, Ciprofloxacin and Moxifloxacin in Human Plasma. Journal of Chromatography B: Analytical Technologies in the Biomedical and Life Sciences, 877, 961-967.

[19] Garces, A., Zerzanova, A., Kucera, R., Barron, D. and Barbosa, J. (2006) Determination of a Series of Quinolones in Pig Plasma Using Solid-Phase Extraction and Liquid Chromatography Coupled with Mass Spectrometric Detection: Application to Pharmacokinetic Studies. Journal of Chromatography A, 1137, 22-29. http://dx.doi.org/10.1016/j.chroma.2006.09.082

[20] Kamberi, M., Tsutsumi, K., Kotegawa, T., Nakamura, K. and Nakano, S. (1998) Determination of Ciprofloxacin in Plasma and Urine by HPLC with Ultraviolet Detection. Clinical Chemistry, 44, 1251-1255.

[21] Krol, G.J., Beck, G.W. and Benham, T.J. (1995) HPLC Analysis of Ciprofloxacin and Ciprofloxacin Metabolites in Body Fluids. Journal of Pharmaceutical and Biomedical Analysis, 14, 181-190. http://dx.doi.org/10.1016/0731-7085(95)01611-2

[22] Maya, M.T., Goncalves, N.J., Silva, N.B. and Morais, J.A. (2001) Simple High-Performance Liquid Chromatographic Assay for the Determination of Ciprofloxacin in Human Plasma with Ultraviolet Detection. Journal of Chromatography B: Biomedical Sciences and Applications, 755, 305-309. http://dx.doi.org/10.1016/S0378-4347(01)00126-8

[23] Sowinski, K.M. and Kays, M.B. (2004) Determination of Ciprofloxacin Concentrations in Human Serum and Urine by HPLC with Ultraviolet and Fluorescence Detection. Journal of Clinical Pharmacy and Therapeutics, 29, 381-387. http://dx.doi.org/10.1111/j.1365-2710.2004.00575.x

[24] Trittler, R., Ehrlich, M., Galla, T.J., Horch, R.E. and Kummerer, K.J. (2002) New and Rapid Fully Automated Method for Determination of Tazobactam and Piperacillin in Fatty Tissue and Serum by Column-Switching Liquid Chromatography. Journal of Chromatography B, 775, 127-132. http://dx.doi.org/10.1016/S1570-0232(02)00298-2 
Scientific Research Publishing (SCIRP) is one of the largest Open Access journal publishers. It is currently publishing more than 200 open access, online, peer-reviewed journals covering a wide range of academic disciplines. SCIRP serves the worldwide academic communities and contributes to the progress and application of science with its publication.

Other selected journals from SCIRP are listed as below. Submit your manuscript to us via either submit@scirp.org or Online Submission Portal.
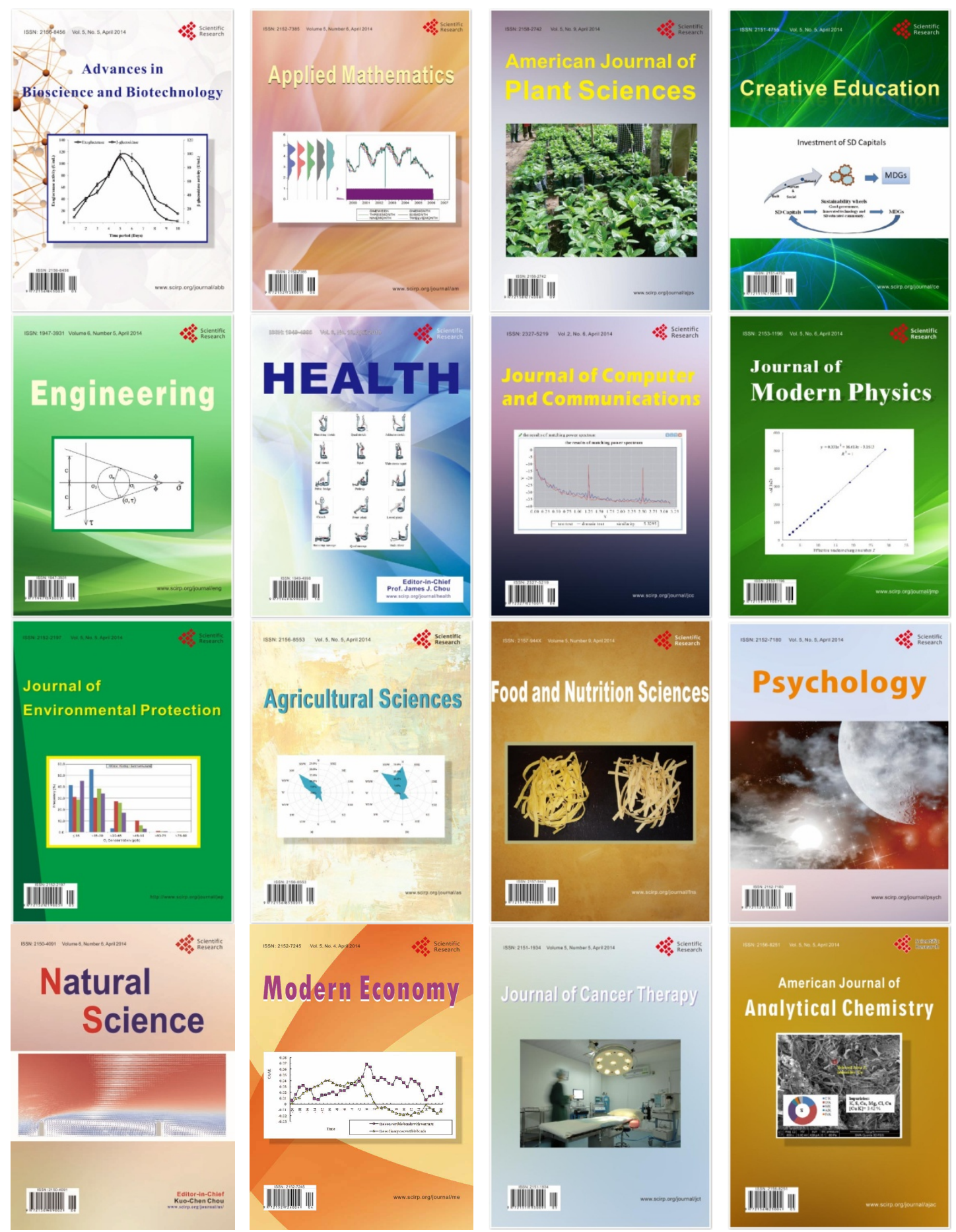